\title{
Controlling a team of unmanned underwater vehicles performing water region search tasks
}

\author{
Andrzej Zak, Ph.D. \\ Polish Naval Academy, Poland
}

\begin{abstract}
The article discusses a problem of performing a mission and controlling a team of unmanned underwater vehicles which perform a task of water region search. The team of underwater vehicles has been modelled as a multi-agent system, which made it possible to use the mechanism of multi-agent systems for controlling the team of cooperating underwater vehicles, with each vehicle being treated as an individual agent. Solutions to cooperation problems in multi-agent systems were proposed, including the presentation of the system structure and the data exchange method. Issues relating to cooperation actions in the team of underwater vehicles were described and solved using the theory of games and the vector quality index. The process of negotiations between the vehicles was modelled as the multiplayer cooperative game, which secured successful realisation of the task performed by the team of vehicles with respect to time, which represents the economic aspect of the task. The optimal solution was selected based on the vector quality index, which provided opportunities for minimisation of costs having the form of energy used by the entire team, and maximisation of profits in the form of time needed for completing the water region search task. The proposed method for dividing the action space in the water region search tasks performed by the team of underwater vehicles consists in assigning to each of cooperating underwater vehicles a task the realisation of which contributes to the realisation of the globally defined task. Finally, the results of water region search tasks performed by a team of cooperating underwater vehicles are presented and discussed taking into account possible vehicle failures happening during task realisation.
\end{abstract}

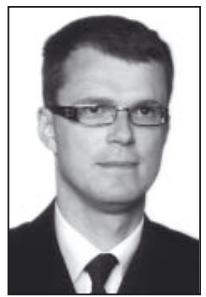

Key words: unmanned underwater vehicles; multi-agent systems; control; multiplayer cooperative games

\section{INTRODUCTION}

Nowadays, research activities concerning generally understood robotics are frequently oriented on the use of teams of cooperating robots performing tasks which cannot be performed, or will be performed less efficiently by a single robot. Such a situation can be observed in underwater robotics where concepts are developed for certain tasks to be performed by a team of underwater vehicles. These tasks most often include exploration and inspection activities, which in general can be done by a single robot but a team of robots can do them faster and more effectively. Another kind of tasks of this type are transport activities in which overall dimensions and the shape of the transported structure make the task to be only done by a team of cooperating vehicles.

A team of cooperating robots reveals numerous advantages, such as high resistance to failures, for instance, which makes it possible to perform the task even if one of robots beaks down. Moreover, in some cases the decomposition of the task to be performed by the team of robots allows us to use robots with simpler structure than that of a single robot performing the same task. However, the problem of controlling the team of cooperating robots is much more difficult than controlling a single vehicle. In case of a team of robots, additional issues are to be discussed, including communication between the vehicles, coordination of actions, performing negotiations, and many others.

The computer sciences make more and more frequent use of multi-agent systems, especially to solve problems of distributed nature, or those requiring complicated calculations. The multiagent system comprises a number of agents which communicate and cooperate with each other to perform a common task. The mechanism of the multi-agent system can be easily adopted to control the team of cooperating underwater vehicles in which each vehicle is treated as an individual agent.

Below presented is a concept of a multi-agent system which was used to perform a task of water region search by a team of cooperating underwater vehicles. The team is assumed to consist of homogeneous and cable-free underwater vehicles equipped with instruments for navigation, technical observation and communication used for data exchange between the vehicles. The underwater vehicles have docking stations situated at one point of the water region, where they can load their internal energy sources, transmit the collected data and receive commands to start work. The shape of the searched water region is defined by the vertexes of the polygon enclosing the region. The water region is divided by the superior system into areas, each of which is to be searched by a single 
underwater vehicle. The information on the area to be searched and the corresponding passing trajectory is obtained by each vehicle from the docking station, together with the order to start work.

\section{AGENT}

The literature on the subject does not deliver any precise definition of an agent. Generally we can say that the agent is an element which is used for creating decentralised systems [2]. Another definition says that the agent is an object which can both observe the environment and act on it [15]. The most precise definition describes the agent as a system situated in a given environment which can make use of certain resources and be able to act autonomously in order to achieve assumed goals $[4,13,14]$. To make the underwater vehicle fulfil the above definition, it has to be equipped with sensors to record input signals coming from the environment, and effectors using which it can act on the environment. Additionally, is should have sort of internal awareness which will secure its autonomy manifesting itself as making decisions on actions to be performed to achieve the assumed goal. We can say that the agent can adapt its behaviour to a current situation. It should be noted, however, that the goal to be performed by one agent can differ from that assigned to another agent, and in case of common task realisation the request coming from the other agent can be realised only when it is in line with the interest of the asked agent.

To describe formally the action of the agent we introduce the following notations: $S=\left\{s_{1}, s_{2}, \ldots\right\}$ represents possible environment states, and $\mathrm{A}=\left\{\mathrm{a}_{1}, \mathrm{a}_{2}, \ldots\right\}$ possible actions of the agent. In this situation the action can be meant as the function $[13,4]$ :

$$
\text { action: } \mathrm{S}^{*} \rightarrow \mathrm{A}
$$

where:

$\mathrm{S}^{*}$ - the selected sequence of environment states.
The environment can be defined as the function:

$$
\text { environment: } \mathrm{S} \times \mathrm{A} \rightarrow \mathrm{S}
$$

The above notations mean that the undertaken action results from the environment state, while the environment modifies its state due to the action of the agent.

We should also define a function which allows the agent to read input signals. This action can be written as the function:

$$
\text { perception: } \mathrm{S} \rightarrow \mathrm{P}
$$

which maps the state of environment into perception P. Consequently, the action function takes the following form:

$$
\text { action: } \mathrm{P}^{*} \rightarrow \mathrm{A}
$$

where:

$\mathrm{P}^{*}$ - the perception sequence.

Assigning the property of autonomy to the agent consists in defining the function of actions which are to be initiated by the agent depending on input signals composing the perception. To do so, we should select a method of implementation of the decision function, at the same time selecting an appropriate type of agent. In case of underwater vehicles it seems a best solution to use the hierarchical layer architecture, the advantages of which include no need to define the decision making mechanism and simplicity of action. A popular sample of this type of architecture is the InterRRaP architecture (Fig. 1) [11, 12]. It consist of a series of layers, the lowest of which is responsible for reflex actions, the middle layer for planning, and the top layer for cooperation with other agents $[4,11,12]$. Each layer has its database which represents the state of environment attributed to this layer. The flow of control in this architecture is realised in two directions: first the control signal is transmitted from lower to higher layers, and then from higher to lower layers. If the lower layer, making use of its database, is not able to take a relevant action, it sends the signal to the higher layer. That means that the higher the layer, the more rarely it is activated.

\section{ENVIRONMENT}

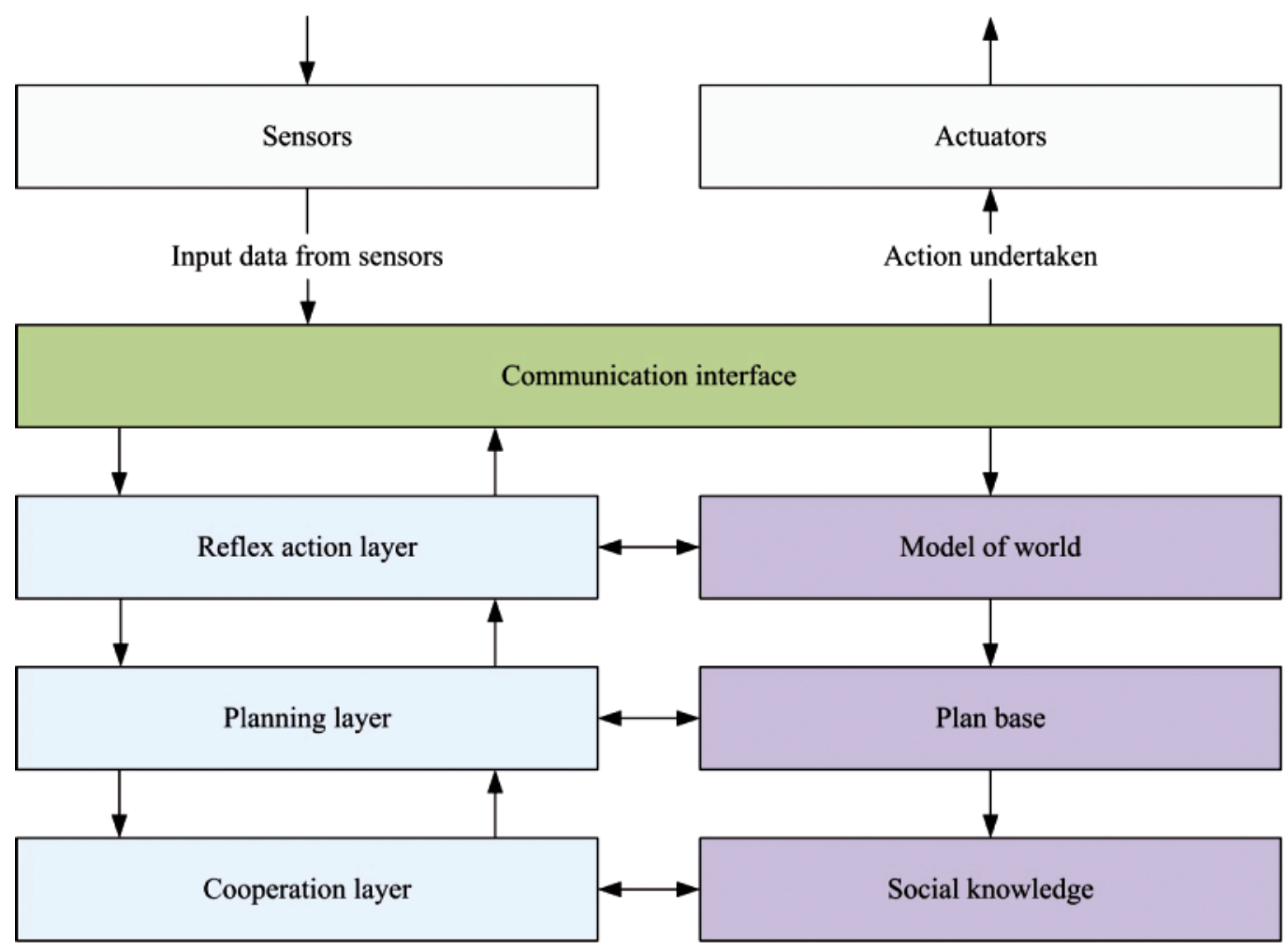

Fig. 1. Flow of control in InteRRaP type architecture [4] 
According to the adopted assumptions, a single underwater vehicle is an agent whose task is to search the water region in cooperation with other vehicles. Therefore the lowest layer of the agent is responsible for avoiding obstacles [17]. The middle layer is responsible for precise motion of the underwater vehicle along the given trajectory [16], while the role of the highest layer is to cooperate with the remaining vehicles in the team.

\section{MULTI-AGENT SYSTEM Structure of the multi-agent system}

A team of cooperating underwater vehicles in which each vehicle is an agent composes a multi-agent system. The agents in this system communicate with each other, at the same time preserving the autonomy of their operation and decision making, which is reflected in actions undertaken by particular agents. Such an interaction between the agents leads to the modification of knowledge and actions performed by individual agents based on the observation of the behaviour of other agents and information transmitted in the communication process. An individual agent is equipped with the strategy leading it to achieving the assumed goal. However, being part of the multiagent system it has to put in practice the strategy of cooperation with other agents, especially when this strategy facilitates reaching the goal common for the entire team. Cooperation and competition between the agents in a multi-agent system is its key feature [9]. To make it possible, we should define the structure and formulate protocols of interaction to provide opportunities for describing message exchange scenarios and allow making decisions.

The multi-agent system can have a hierarchic structure, in which a decision about future actions is made by the team leader, or a flat structure, in which all agents are equal and decisions are made as a result of negotiations. The advantage of the hierarchical structure is high speed of decision making, as the time-consuming process of negotiations can be avoided here. Its disadvantage is relatively poor use of all team potential, as the team leader can make decisions based on the information on the environment and other agents which is insufficient to make the right decision. The problem also arises when the current leader breaks down and mechanisms of selection of a new leader are to be applied. In case of flat structure this problem does not exist and decisions are made collectively. Unfortunately, decision making in this structure is a very time consuming process.

Either of these two structures can be successfully used in the task of water region search realised by a team of autonomous underwater vehicles. However, since the water environment is very dangerous and unfriendly to technical equipment, we should assume that failures of vehicles can happed relatively often. Moreover, search tasks can be performed in relatively long times, which means no limits concerning the speed of decision making processes. That is why the flat structure seems more appropriate and better promises success in the realisation of tasks of the above type.

\section{Data exchange}

The essence of the multi-agent system is coherent behaviour of all agents composing the team. Difficulties in the realisation of this task result from the fact that multi-agent systems have no central control, and securing coherent action of particular agents requires data exchange via mutual communication. Therefore we should assume that each agent has ability to send, receive an analyse the data, and that there is a well-defined protocol of interaction, the same for all agents.
The interaction protocols are divided, in general, into coordination protocols and communication protocols. The coordination protocols are used for periodical exchange of information on current condition of particular agents, including redundancy avoiding, while the cooperation protocols are used for settling cooperation between particular agents, i.e. task decomposition and distribution. The action of both protocols requires a relevant mechanism of data exchange. An interesting solution in this context seems to be the interaction mechanism of Blackboard type, the idea of which consists in the existence of common memory, frequently bearing the name of a blackboard, in which agents can write and read data. This way each agent has access to the data recorded by other agents and can, at an arbitrary time, write and read data in the blackboard. The data stored in the blackboard are valid for certain time, or until they are updated by more current data. Since the adopted structu re is flat without a defined leader which would be responsible for data storage, the common memory will be stored by each agent. In other words, each agent will have a copy of the information stored in the blackboard. In this case data modification in the blackboard will consist in transmitting new data by one agent to all remaining agents, after which the data will be updated in the blackboard.

Since the underwater vehicles perform their tasks in the water environment, the common transmission medium of all agents is water, and the transmitted messages will have the form of mechanical (sound) waves. In this context it seems reasonable to use a token ring mechanism, which was successfully used in computer networks. The action of this data exchange mechanism makes use of the exchange of authorisation, or a so-called token. The vehicles are numbered in accordance with the increased distance of the area to be searched by them from the docking place. The agents, one by one, receive a token which allows them to pass a message, and then pass the token over to the next vehicle. The last vehicle passes the token to the first one. The agent which wants to pass the message waits for a free token, and having received it changes it into the occupied token and passes the data block bearing the name of a frame.

\section{Cooperative action}

The cooperative action in multi-agent systems can be described as a multiplayer cooperative game. The cooperative game will be an ordered pair having the form [1]:

$$
\Gamma=(\mathrm{N}, \mathrm{v})
$$

where:

$\mathrm{N}=\{1, \ldots, \mathrm{n}, \ldots, \mathrm{N}\}-$ the set of player numbers $\mathrm{v}: 2^{\mathrm{N}} \rightarrow \Re^{2} \quad-$ the characteristic function of the game.

The characteristic function of the game assigns to each set $\mathrm{S} \subset \mathrm{N}$, where $\mathrm{S}$ represents all possible coalitions in the team of agents including those composed of single agents, a total maximal secured payment $v(S)$ which can be obtained by the members of coalition $\mathrm{S}$ irrespective of the action undertaken by the remaining players. It is additionally assumed that $v(\varnothing)=0$ and $v(\cdot)$ is super-additive i.e.:

$$
v(\mathrm{~S})+v(\mathrm{~T}) \leq v(\mathrm{~S} \cup \mathrm{T}) ; \mathrm{S}, \mathrm{T} \subset \mathrm{N} ; \mathrm{S} \cap \mathrm{T}=\varnothing
$$

A solution to the multiplayer cooperative game can be presented as the solution to the multi-criteria optimisation of the following type:

$$
(\mathrm{X}, \mathrm{F}, \mathrm{R})
$$

where:

$\mathrm{X}-$ the nonempty set of feasible solutions,

$\mathrm{F}: \mathrm{X} \rightarrow \mathfrak{R}^{\mathrm{N}}-$ the vector quality index, and $\mathrm{R}$ defines the relation of domination. 
When analysing the cooperation between the agents performing the task of water region search, the set of feasible solutions is the set of all possible coalitions which can be formed between these agents. The criterion function assigns to each feasible solution $\mathrm{x} \in \mathrm{X}$ its numerical valuation, important for selecting the best solution. In search-type tasks we tend to minimise the time of task realisation, which is directly connected with the minimisation of the length of the path to be covered by each vehicle. Since the cooperation game is played when one of agents cannot do the assigned task and another agent, or coalition of agents, is to take it over, we will want the profit from the task done by this vehicle or coalition of vehicles to be the maximal, the cost borne by each vehicle to perform the supplementary work to be the minimal, and the coalition performing the supplementary work to be the largest. The above assumptions would allow the team of vehicles to do the water region search task at the shortest possible time. Consequently, the quality index can be defined as:

$$
\mathrm{F}(\mathrm{x})=\{\mathrm{Z}, \mathrm{K}, \mathrm{L}\}
$$

where:

Z - the profit which can be obtained as a result of supplementary action undertaken by the coalition of agents,

$\mathrm{K}$ - the cost borne by each agent composing the coalition formed to do the supplementary work,

L - the number of coalition members.

Let $\mathrm{W}_{\mathrm{D}}$ represent the cost which a given agent has to bear to reach the place from which the supplementary action begins. This cost can be identified with the length of the path between the last point of its own trajectory and the point from which the supplementary action begins. Additionally, let $\mathrm{W}_{\mathrm{T}}$ represent the cost connected with covering the remaining part of the trajectory within the framework of the realisation of the supplementary task, which can be identified with the length of the trajectory to be covered in this supplementary action. In this case the $\operatorname{cost} \mathrm{K}_{\max }$ is the maximal cost borne by a single vehicle performing the supplementary task with the longest approach path, which can be written as:

$$
\mathrm{K}_{\max }=\mathrm{W}_{\mathrm{T}}+\max _{\mathrm{i} \in \mathrm{N}}\left(\mathrm{W}_{\mathrm{D}, \mathrm{i}}\right)
$$

where:

i - the agent number from within a set of all efficient agents.

When a coalition between a number of agents is formed, the task will be performed simultaneously by all coalition members. Therefore the cost borne by a single agent composing the coalition will be the sum of the cost borne for realisation of the remaining trajectory part and the cost of approach, taking into account the number of agents composing the coalition, which can be written as:

$$
\mathrm{K}=\frac{\mathrm{W}_{\mathrm{T}}+\sum_{\mathrm{i} \in \mathrm{S}} \mathrm{W}_{\mathrm{D}, \mathrm{i}}}{\mathrm{L}}
$$

The profit $\mathrm{Z}$ obtained by the given coalition of agents will be defined as:

$$
\mathrm{Z}=\mathrm{K}_{\max }-\mathrm{K}
$$

which means that it is the difference between the maximal cost of realisation of the supplementary work by a single agent with the longest approach path and the cost to be borne by the agent as a coalition member.
A set of all values of the criterion function $F$ which it takes over a set $\mathrm{X}$ creates the criteria space $\mathrm{Y}$ :

$$
\mathrm{Y}=\left\{\mathrm{y}=\mathrm{F}(\mathrm{x}) \in \mathfrak{R}^{\mathrm{N}} \mid \mathrm{x} \in \mathrm{X}\right\}
$$

being the map of the set $\mathrm{X}$ in $\mathfrak{R}$.

To select a desired solution $\mathrm{x}$ from the set $\mathrm{X}$ we have to compare different solutions. This comparison is realised by comparing numerical values of particular estimations $\mathrm{F}_{\mathrm{n}}(\mathrm{x})$ attributed to the compared solutions. Therefore we can say that the decision whether solution $\mathrm{x}_{1}$ is better than solution $\mathrm{x}_{2}$ is made by comparing their numerical images $\mathrm{F}\left(\mathrm{x}_{1}\right)$ and $\mathrm{F}\left(\mathrm{x}_{2}\right)$. We should define the relation $\mathrm{R} \subset \mathrm{Y} \times \mathrm{Y}$, i.e. the relation of domination which will make it possible to decide whether the estimation of the image $F\left(x_{1}\right)=y_{1}$ is better than that of $F\left(x_{2}\right)=y_{2}$, or not.

The relation $\mathrm{R}$ can be defined using the propositional function in the following way:

$$
\mathrm{R}_{\varphi}=\left\{\left(\mathrm{y}_{1}, \mathrm{y}_{2}\right) \in \mathrm{Y} \times \mathrm{Y} \mid \varphi\left(\mathrm{y}_{1}, \mathrm{y}_{2}\right)=1\right\}
$$

where the propositional function $\varphi: \mathrm{Y} \times \mathrm{Y} \rightarrow\{0,1\}$ has the form:

$$
\varphi\left(\mathrm{y}_{1}, \mathrm{y}_{2}\right) \equiv \mathrm{y}_{1} \text { is better than } \mathrm{y}_{2}
$$

In the analysed case, for the quality index having the form of (3.16) the relation of domination can be defined in the following way:

$$
\begin{gathered}
\varphi\left(\mathrm{y}_{1}, \mathrm{y}_{2}\right) \equiv \mathrm{L}_{1} \geq \mathrm{L}_{2} \\
\varphi\left(\mathrm{y}_{1}, \mathrm{y}_{2}\right) \equiv \mathrm{Z}_{1} \geq \mathrm{Z}_{2} \\
\text { and } \\
\mathrm{Z}_{1} \geq\left|\mathrm{L}_{1}\right| \max _{\mathrm{n} \in \mathrm{S}_{1}} \mathrm{Z}(\{\mathrm{n}\}) \wedge \mathrm{Z}_{2} \geq\left|\mathrm{L}_{2}\right| \max _{\mathrm{n} \in \mathrm{S}_{2}} \mathrm{Z}(\{\mathrm{n}\}) \\
\varphi\left(\mathrm{y}_{1}, \mathrm{y}_{2}\right) \equiv \mathrm{K}_{1} \leq \mathrm{K}_{2} \\
\text { and } \\
\mathrm{K}_{1, \mathrm{i}}-\mathrm{W}_{1, \mathrm{D}, \mathrm{i}} \geq 2 \mathrm{~W}_{1, \mathrm{D}, \mathrm{i}} \wedge \mathrm{K}_{2, \mathrm{i}}-\mathrm{W}_{2, \mathrm{D}, \mathrm{i}} \geq 2 \mathrm{~W}_{2, \mathrm{D}, \mathrm{i}}
\end{gathered}
$$

Condition (15) says that the coalition performing the supplementary task is to be as large as possible. Condition (16) says that the profit to be obtained as a result of coalition formation is to be the maximal, and at least L times as big as the maximal profit of all coalition members, where $\mathrm{L}$ is the number of coalition members. Condition (17) says that each coalition member is to have an attributed search trajectory with the length larger or at least equal to two lengths of the approach path to the point at which the supplementary action starts.

\section{DIVISION OF ACTION SPACE}

When a team of cooperating underwater vehicles performs a task, a problem which is to be decided upon is how to divide the action space. The division of the action space consists in allocating an individual task to each of cooperating underwater vehicles is such a way that all individual tasks compose the globally defined task. In case of water region search task performed by a number of underwater vehicles, the division of the action space will consist in physical separation of water region areas to be searched by a single underwater vehicle. Searching all areas of the water region divided in the above way will be equivalent with the realisation of the search of the entire water region. 
A solution to this problem consists in geometrical division of the water region treated as a polygon into smaller polygons, being separate areas for which probing lines and underwater vehicle passing trajectories are then determined $[3,7,8,10]$. However, this solution has some disadvantages. For instance, in this solution the time needed for reaching the searched area by the underwater vehicle, a parameter of certain importance, cannot be estimated. Moreover, when determining probing lines and passing trajectories for each of the separated areas, it may happen at the border between the adjoining areas that some area parts are omitted, or are searched twice or more times. These disadvantages can be eliminated by introducing another method of action space division, which consists in determining first the probing lines and passing trajectories for the entire searched water region, and then dividing the trajectories into segments, each of which to be covered by a single underwater vehicle. Such a solution has certain advantages, the most important of which is elimination of time-consuming procedures of geometrical division of the water region and substituting it by a simple iterative algorithm of trajectory division into segments. Moreover, after combining together, the data recorded by all underwater vehicles performing the water region search task have the same form as those obtained, hypothetically, by a single underwater vehicle. The proposed solution also secures uniform distribution of work over all underwater vehicles and provides opportunities for covering the entire area of the water region by technical observation means without multiplying the observation. Figure 2 shows differences between dividing the action space on the geometrical basis and using the passing trajectory division.

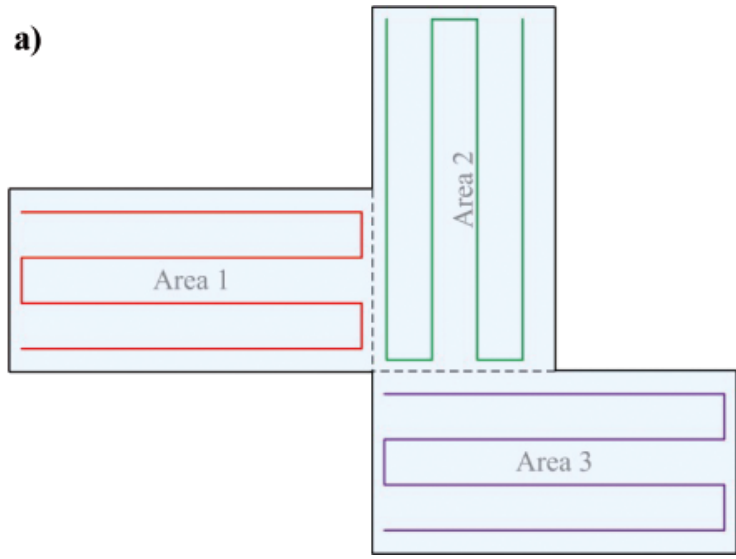

b)$$
\text { Area } 3
$$

Fig. 2. Division of action space: a) geometrical division of water region, b) division of passing trajectory.

The basic idea of the proposed solution consists in dividing the full trajectory worked out for the entire searched water region into segment explored by particular underwater vehicles. In order to meet the condition that all underwater vehicles taking part in the search are similarly loaded the trajectory is to be divided in such a way that each segment has approximately the same length, including the paths of vehicle approach to the starting point of search operation and return to the docking place.

The task consisting in trajectory division into segments can be defined in the following way: let $\mathrm{n} \geq 2$ represent the number of segments into which the trajectory $\overline{\mathrm{SK}}$ is to be divided. We assume the existence of such points $\mathrm{A}_{i}$ belonging to the trajectory $\overline{\mathrm{SK}}$ that:

$$
\left|\overline{\mathrm{SA}_{1}}\right|+\sum_{\mathrm{i}=1 \ldots \mathrm{n}-2}\left|\overline{\mathrm{A}_{\mathrm{i}} \mathrm{A}_{\mathrm{i}+1}}\right|+\left|\overline{\mathrm{A}_{\mathrm{n}-1} \mathrm{~K}}\right|=|\overline{\mathrm{SK}}|
$$

and that

$$
\begin{gathered}
\left|\overline{\mathrm{SA}_{1}}\right|+\mathrm{d}_{0}=\left|\overline{\mathrm{A}_{1} \mathrm{~A}_{2}}\right|+\mathrm{d}_{1}= \\
=\ldots=\left|\overline{\mathrm{A}_{\mathrm{i}} \mathrm{A}_{\mathrm{i}+1}}\right|+\mathrm{d}_{\mathrm{i}}=\ldots=\left|\overline{\mathrm{A}_{\mathrm{n}-1} \mathrm{~K}}\right|+\mathrm{d}_{\mathrm{n}-1}
\end{gathered}
$$

where: $d_{i}$ is the total length of the shortest path of approach to point $A_{i}$ and return from point $A_{i+1}$, i.e.:

$$
\mathrm{d}_{\mathrm{i}}=\left|\overline{\mathrm{SA}_{\mathrm{i}}}\right|+\left|\overline{\mathrm{A}_{\mathrm{i}+1} \mathrm{~S}}\right|
$$

Condition (18) secures that the entire trajectory will be used when dividing the trajectory into segments, while condition (19) means that each trajectory segment, together with the approach and return paths, will have the same length.

\section{RESULTS OF INVESTIGATIONS}

In previous sections a solutions was proposed which allows controlling a team of underwater vehicles cooperating within the framework of the multi-agent system. The control algorithms making use of the multi-agent system and combined with the algorithm of control along a given trajectory 516 were implemented on the computer, which provided opportunities for performing their tests.

As a test sample, the task of Gdynia harbour search was performed by a team consisting of eight cooperating underwater vehicles. It was assumed in the test that the underwater vehicle 2 broke down after covering 3947.6 [m] of the trajectory attributed to it. According to the adopted solution the remaining underwater vehicles started negotiations to settle which vehicle, or coalition of vehicles will complete the task started by vehicle 2. After negotiations, the vehicles presented costs of realisation of the supplementary task, see table 1 .

As a result of the performed negotiations, profits from particular coalitions were calculated, and then coalitions securing highest profits were selected in groups of different numbers of coalition members (table 2). It was checked for each coalition whether the conditions (15) (16) and (17) are met. Based on that it was concluded that the most profitable solution will take place when the supplementary task is taken over by a coalition of agents consisting of vehicle 3 and vehicle 5. Since the coalition consists of two members the profit will be 2.96 times as high as in case of single vehicle coalition, while the cost of search will be 5.75 times as high, compared to the cost of approach of vehicle 3, and 3.76 times as high as for vehicle 5. In case of larger coalitions, condition (17) is not met for one or more vehicles. For coalitions composed of more than 3 vehicles condition (16) is not met either. Table 3 collects the numerical data describing the performed task, while Figure 3 shows the passing trajectories of each underwater vehicle against the electronic map of the searched water region. 
Tab. 1. Presented costs of supplementary action to be undertaken after failure of vehicle 2

\begin{tabular}{|c|c|c|c|c|c|c|c|}
\hline Vehicle number & 1 & 3 & 4 & 5 & 6 & 7 & 8 \\
\hline Presented cost & 1100.6 & 526.4 & 1042.9 & 769.2 & 1404.1 & 1417.3 & 1942.7 \\
\hline
\end{tabular}

Tab. 2. Data settled in the negotiation process after vehicle 2 break-down

\begin{tabular}{|c|c|c|}
\hline Coalition & Profit & Cost borne by a single vehicle \\
\hline$\{3\}$ & 1416.3 & 6336.9 \\
\hline$\{3,5\}$ & 4200.15 & 3553.0 \\
\hline$\{3,4,5\}$ & 5036.9 & 2716.3 \\
\hline$\{1,3,4,5\}$ & 5440.8 & 2312.4 \\
\hline$\{1,3,4,5,6\}$ & 5622.5 & 2130.74 \\
\hline$\{1,3,4,5,6,7\}$ & 5741.4 & 2011.8 \\
\hline$\{1,3,4,5,6,7,8\}$ & 5751.2 & 2002.0 \\
\hline
\end{tabular}

Tab. 3. Data of the performed task of Gdynia harbour search

\begin{tabular}{|c|c|c|c|c|}
\hline Harbour area $\left[\mathrm{m}^{2}\right]$ : & 1714708.2 & \multicolumn{2}{|c|}{ Number of vehicles in the team [-]: } & 8 \\
\hline \multirow{2}{*}{ Shoreline length $[\mathrm{m}]$ : } & \multirow{2}{*}{15334.7} & \multirow{2}{*}{ Vehicle 1: } & path length $[\mathrm{m}]:$ & 9676.2 \\
\hline & & & realisation time $[\mathrm{min}]$ : & 80.65 \\
\hline \multirow{2}{*}{ Water region depth $[\mathrm{m}]$ : } & \multirow{2}{*}{16.0} & \multirow{2}{*}{ Vehicle 2: } & path length $[\mathrm{m}]$ : & 3947.6 \\
\hline & & & realisation time [min]: & 33.0 \\
\hline \multirow{2}{*}{$\begin{array}{l}\text { Distance from bottom when } \\
\text { performing the task }[\mathrm{m}]:\end{array}$} & \multirow{2}{*}{13.0} & \multirow{2}{*}{ Vehicle 3: } & path length $[\mathrm{m}]$ : & 12640.8 \\
\hline & & & realisation time $[\mathrm{min}]$ : & 105.4 \\
\hline \multirow{2}{*}{ Observation zone width $[\mathrm{m}]$ : } & \multirow{2}{*}{55.8} & \multirow{2}{*}{ Vehicle 4: } & path length $[\mathrm{m}]$ : & 9867.1 \\
\hline & & & realisation time [min]: & 82.5 \\
\hline \multirow{2}{*}{$\begin{array}{c}\text { Distance between probing } \\
\text { lines }[\mathrm{m}]:\end{array}$} & \multirow{2}{*}{54.4} & \multirow{2}{*}{ Vehicle 5: } & path length $[\mathrm{m}]$ : & 13044.1 \\
\hline & & & realisation time [min]: & 108.7 \\
\hline \multirow{2}{*}{ Course of probing lines: } & \multirow{2}{*}{$80^{\circ}$} & \multirow{2}{*}{ Vehicle 6: } & path length $[\mathrm{m}]$ : & 9437.1 \\
\hline & & & realisation time [min]: & 78.7 \\
\hline \multirow{2}{*}{ Total path length $[\mathrm{m}]$ : } & \multirow{2}{*}{42494.4} & \multirow{2}{*}{ Vehicle 7: } & path length $[\mathrm{m}]$ : & 9647.7 \\
\hline & & & realisation time [min]: & 80.4 \\
\hline \multirow{2}{*}{$\begin{array}{l}\text { Total time of task realisation } \\
\text { by a single vehicle [min]: }\end{array}$} & \multirow{2}{*}{354.12} & \multirow{2}{*}{ Vehicle 8: } & path length $[\mathrm{m}]:$ & 9879.3 \\
\hline & & & realisation time [min]: & 82.5 \\
\hline
\end{tabular}

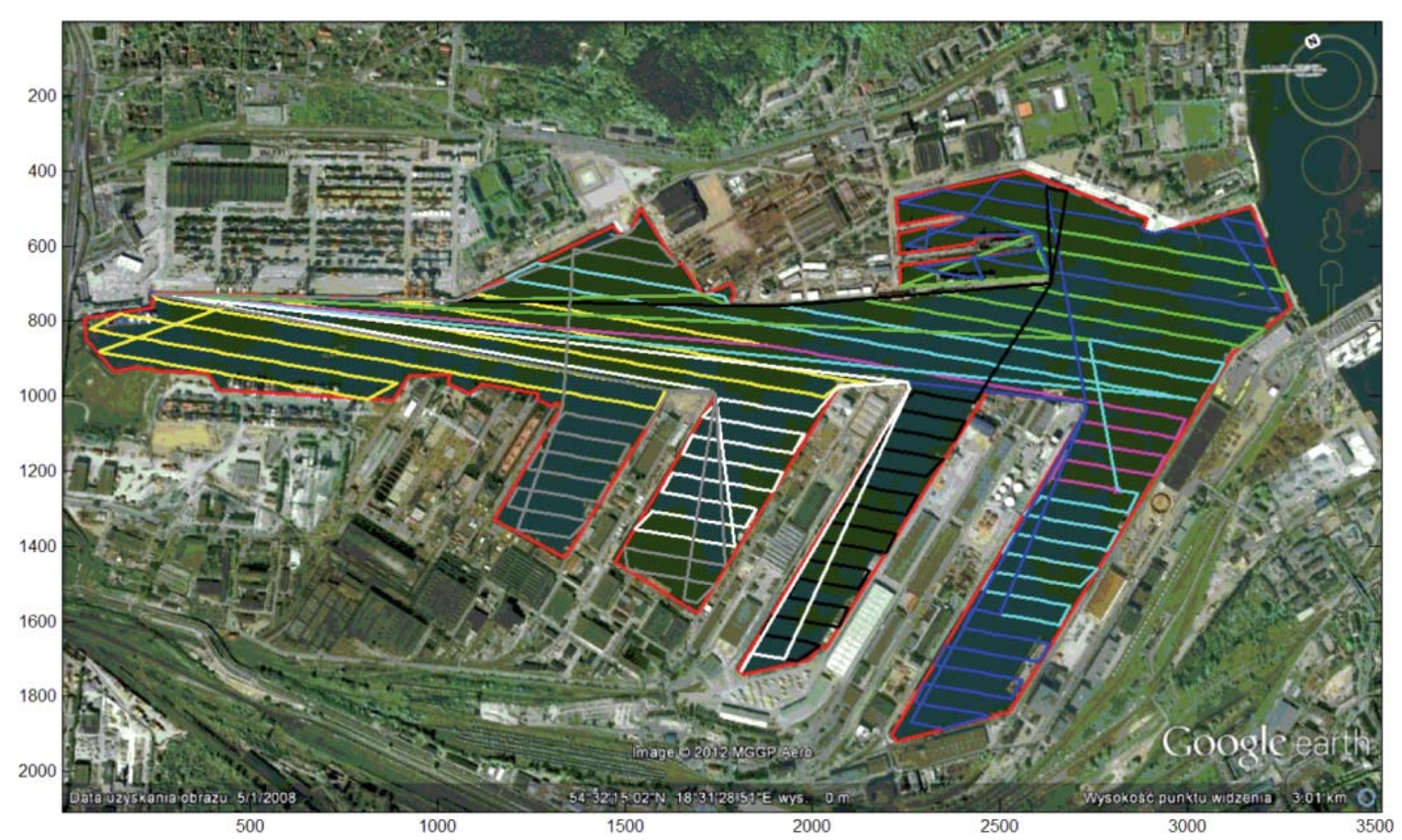

Fig. 3. Passing trajectories realised by each of underwater vehicles composing the team which performed the task of Gdynia harbour search 
Based on the performed tests we can conclude that the use of many vehicles for searching a water region leads to considerable reduction of time needed for doing this task. It is noteworthy, however, that this time does not decrease proportionally to the number of the used vehicles, which results from the fact that the time of approach of each vehicle to the area of its operation is to be taken into account. Assuming no vehicle break-down, a single vehicle needs 129.2 [min] for covering the trajectory having the length of 15503.9 [m], while the cooperation of five underwater vehicles extends the trajectory by 7623.6 [m], which is the total approaching distance of the vehicles to the area of operation. However, at the same time the total searching time is 3.3 time shorter and is reduced to 39.4 [min]. Assuming that one vehicle breaks down during task realisation, a single vehicle needs 354.12 [min] for covering the trajectory having the length of 42494.4 [m], while the team of eight cooperating vehicles extends this distance nearly twice, to $78139.9[\mathrm{~m}]$, but the time of task realisation is equal to 108.7 [min], i.e. 3.3 times faster than it would be done by a single underwater vehicle. Another advantage of coalition which should be mentioned here is that the search task for the entire water region can be successfully completed, which would not be possible in case of one vehicle performing the search and its subsequent failure during task realisation.

\section{CONCLUSIONS}

Controlling a team of autonomous underwater vehicles is a complicated issue, especially in case when the motion of vehicles is subject to limitations oriented on minimisation of the task realisation time. The problem becomes even more difficult when the vehicles have to cooperate to perform supplementary tasks after a failure of one or more vehicles taking part in the search action. Taking into account water as unfriendly environment and difficulties connected with precise realisation of the set trajectory, as well as with navigation and communication, we should conclude that the problem of controlling the team of underwater vehicles is extremely nontrivial. Its solution needs an untypical combined approach, which will link the knowledge from within the fields of automation, computer science, communication, and sociology. All these features can be found in multi-agent systems which are still in the phase of tests an definition of principles. Their basic idea secures cooperation of a team of robots acting to achieve a common goal which is the realisation of the formulated task.

The performed tests have revealed that controlling the team of underwater vehicles with the aid of a multi-agent system provides opportunities for efficient realisation of the task defined as searching a given water region. Adopting the above concept to the underwater search task resulted in doing the task in much shorter time than in case of a single vehicle performing the same task, simultaneously securing higher chances of success of the mission even when one or more underwater vehicles break down. The proposed solutions permit more efficient distribution of individual tasks, being parts of the global task, over individual vehicles composing the team. The proposed communication solutions make it possible to exchange efficiently the information between the agents, which secures fluent realisation of both coordination and cooperation actions. Moreover, defining the cooperative actions as the multiplayer cooperative game with vector criteria function allows us to use multi-criteria optimisation to solve problems of supplementary actions to be undertaken after failure of one or more agents. The adopted quality indices and the defined functions of domination secure the realisation of the water region search task by a team of underwater vehicles in minimal time and at minimal costs. The proposed algorithms of agent's operation along with the defined actions of cooperation performed during the negotiations secure proper behaviour of individual agents and, consequently, of the entire team.

It is noteworthy that the combined use of a number of cooperating underwater vehicles in water region search tasks result in shortening the time needed for doing the task. However, the time reduction is not proportional to the increasing number of the used vehicles, which is connected with extra time expenses to be covered by each vehicle to reach the area of its action. Taking this into account when dividing the action space makes it possible to assign optimally partial tasks, while at the stage of cooperation actions it allows to choose the coalition of vehicles performing the supplementary action in such a way that both the time of operation of the entire team and cost of the supplementary operation are minimal. The adopted solutions make it possible to determine the minimal time needed for performing the search action by the team of underwater vehicles in the given water region, even after assuming the failure of one or more vehicles before the start of the search. Moreover the proposed solutions make it possible to determine the optimal number of underwater vehicles which will perform the action in the given water region, thus minimising both the time of search and the number of vehicles.

\section{REFERENCES}

1. Ameljańczyk A.: Multi-criteria optimisation in control and management problems, (in Polish), Zakład Narodowy im. Ossolińskich, 1984

2. Cetnarowicz K., Gruer P., Hilaire V., Koukam A.: A Formal Specification of M-Agent Architecture, Revised Paper from the Second International Workshop of Central and Eastern Europe on Multi-Agent Systems, Springer-Verlag, London UK, 2002, pp. $62-72$

3. Choset H.: Coverage for robotics - A survey of recent results, Annals of Mathematics and Artificial Intelligence 31, Kluwer Academic Publishers, 2011, pp. 113-126

4. Ferber J.: Multi-Agents Systems. An Introduction to Distributed Artificial Intelligence, Addison Wesley, London 1999

5. Garus J., Żak B.: Using of soft computing techniques to control of underwater robot, 15th International Conference on Methods and Models in Automation and Robotics (MMAR), 2010, pp. $415-419$

6. Gnatowski M.: Application of multi-agent systems in cooperation of mobile robots (in Polish), Ph.D. thesis, Institute of Fundamental Technological Research PASN, Warsaw 2005.

7. Hert S., Lemelsky V.: Polygon Area Decomposition for Multiple-Robot Workspace Division, International Journal of Computational Geometry and Application, vol. 8, no. 4, 1998, pp. $437-466$

8. Hert S., Tiwari S., Lumelsky V.: A Terrain-Covering Algorithm for an $A U V$, Underwater Robots, Kluwer Academic Publisher, Boston, 1996, pp. 17-45

9. Łuszpaj A.: JADE Java Agent Development Framework, Internet page: http://home.agh.edu.pl/ kozlak/SA/jade2/jade.htm, access: 10.07.2012

10.Maza I., Ollero A.: Multiple UAV cooperative searching operation using polygon area decomposition and efficient coverage algorithms, Distributed Autonomous Robotic Systems 6, 2007, pp. 221-230

11.Muller J.P., Pischel M.: The Agent Architecture InteRRaP. Concept and Application, German Research Center for Artificial Intelligence, 1993

12.Muller J.P.: The Design of Intelligent Agents. A Layered Approach, Lecture Notes in Computer Science, Volume 1177/ 1996, 1996

13.Weiss G.: Multiagent Systems. A Modern Approach to Distributed Artificial Intelligence, The MIT Press, 1999 
14. Wooldridge M.: Jennings N. R.: Intelligent Agents. Theory and Practice, The Knowledge Engineering Review 10 No 2, 1995, pp. $115-152$

15.Zieliński C.: Formal Approach to the Design of Control Software for Assistive Robots in Advanced Manufacturing Systems, Seminar on Human-Robot Collaboration in Manufacturing, Singapore Institute of Manufacturing Technology, SIMTech 2005

16.Żak A.: Trajectory-Tracking Control of Underwater Vehicles, Solid State Phenomena Vol. 196 (2013), Trans Tech Publications Ltd. Switzerland, 2013, pp. 156-165

17.Żak B.: The minimal-time control in collision situation on the sea, Proceedings of the 6th WSEAS International Conference on Systems Theory \& Scientific Computation, 2006, pp. 175-180

\section{CONTACT WITH THE AUTHOR}

Andrzej Zak, Ph.D., Polish Naval Academy ul. Smidowicza 69, 81-103 Gdynia, POLAND e-mail: a.zak@amw.gdynia.pl mobile: +48 691999318 